$\xi=-1$

\title{
Electro arthrography: Recording Electrical Potentials At the Elbow Joint
}

\author{
Sindu Divakaran*, Bethanney Janney J, Priyanga R, Sindoora Jalagam, Ramya.R.C, Shakthi.R \\ Department of Biomedical Engineering, School of Bio and Chemical Engineering, \\ Sathyabama Institute of Science and Technology, Chennai \\ *Corresponding author E-mail: sindudiva@gmail.com
}

\begin{abstract}
Electroarthrography (EAG) is a recent technology proposed to detect cartilage degradation. It is used to record electrical potentials generated on the surface of elbow while the joint undergoes compressive loading. The aim of this work is to understand the generation of EAG signals and to differentiate between the healthy and unhealthy elbow based on its cartilage degradation. It has been found that EAG signals originate from the cartilages based on the simulated and measured potential values distributions. Unhealthy cartilages produce varying current density with specific potential distribution. EAG signals can be used as a clinical tool to diagnose bone and cartilage degradation.
\end{abstract}

Keywords: Cartilage Degradation;Elbow; Electroarthography (EAG)

\section{Introduction}

Cartilage seen at the joints provides cushioning between bones and helps to reduce the friction[1][2]. Damage to the cartilage due to injury or gradual wear and tear, leads to pain, inflammation, stiffness and disability. The damage can be hard to detect through $\mathrm{x}$-rays, ultrasound etc. A method was explored to detect this deterioration in early stages by electroarthography [3]. Electroarthrography is a method in which small electrodes are attached to the skin to detect minute variations in electrical signals that are emitted when cartilage bears weight [6]. This technique is capable of measuring cartilage streaming potentials non-invasively through electrodes contacting the skin surrounding diarthrodial joints[8]. With the help of EAG technique one can assess the articular cartilage degradation at their early stages and can also diagnose the same. The cartilage degradation cannot be found out in $\mathrm{x}$-rays or ultrasound[5].

The main aim of this study is to identify and process signals between healthy and unhealthy subjects and to differentiate the level of cartilage degradation in elbow joint based on these signals. While the existing system identifies the signal at the knee, this model is used to detect the cartilage degradation at the surface of the elbow. It provides an easy way to detect the cartilage degradation that is accurate, reliable and cost effective which can be implemented in hospitals for painless detection. This earlier detection can prevent more deterioration of cartilage caused by trauma or overloading which may result in abnormal biomechanics and some diseases like arthritis, osteoporosis, bone tumor, osteomalacia, etc.

\section{Related Works}

Quingyi Han, et al., 2014, the work deals with understanding of the generation of the EAG signals using computer model. Computer modeling helps us to understand the generation of EAG signals given the structural and electrophysiological complexity of the knee and of the various factors that affect the EAG signals so as to help develop the EAG technology as a useful clinical tool.[9] A.M. Preville et al, 2013., in this paper the technique called electroarthrography (EAG) measures electrical potentials at the surface of the knee during joint loading is described. EAG signals were recorded at eight electrode sites over one knee as the subjects moved their weight from one leg to the other to achieve joint loading. The EAG signals were filtered, baseline-corrected and timeaveraged. These sites also showed the highest mean EAG values. The mean EAG potentials of the control group were significantly higher compared with the OA group for three sites overlying the joint line. This work tells that EAG signals are obtained from the streaming potentials in compressed articular cartilage which are the known sensitive indicators of joint cartilage health.[7]

\section{Materials and Methods}

\subsection{Hardware design}

\subsubsection{Arduino Board}

Arduino is an electronics prototyping platform which is very simple to use. It has an easy to use hardware and software. Instructions are given to the board using arduino programming language and arduino software that is based on processing. They are used in thousands of applications [4]from simple to complicated projects. 


\subsubsection{Piezo Electric Sensor}

The principle of working of a piezo sensor is the conversion of mechanical energy to electrical energy. The output or voltage obtained gives the value of the force or pressure applied. They measure dynamic or changing events. They are used in many pressure sensing applications.

\subsubsection{Resistor}

Resistors are electronic components that controls or limits the flow of current in electrical and electronic circuits. They find their applications to control the same in a wide variety of circuits.

\subsubsection{Bread Board}

Breadboard is an electronic solderless board for giving temporary connections. It is a completely reusable board where soldering is ot required. Required wires and connections are given by inserting them into the holes in the breadboard.

\subsection{Software Design}

The software used for extraction and processing of signal is "Processing 2.0.1". Sophisticated visual structures can be designed using this processing language. Sketches are the programs written using Processing. (Fig 1) The arduino (UNO) and Processing 2.0.1 software is been implemented to process the signals and datalog them in Excel sheet by sketching it in Processor with the Arduino Board.

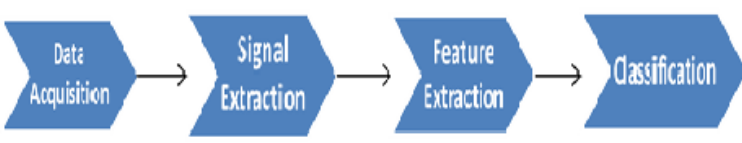

Fig 1: Software Design

\subsection{Methodology}

The basic block diagram of the methodology is given below.(Fig 2)
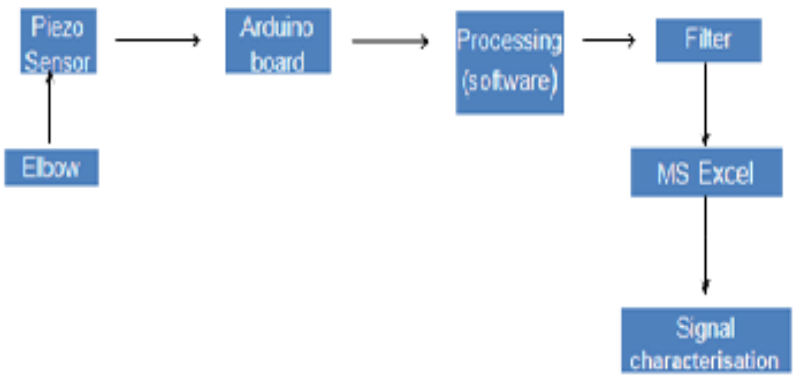

Fig 2: Block diagram

The signals are obtained in milli volts $(\mathrm{mV})$ from four piezosensors placed at the lateral and the medial site of the elbow joint.(Fig 3)

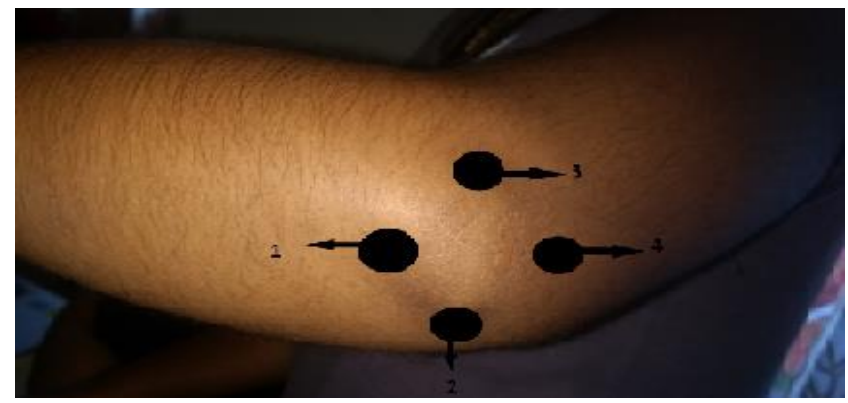

Fig 3: Positioning of piezosensors
The signals are generated for every 10 seconds at the time interval of 120 seconds when a slight movement is given. The obtained signals are later averaged in each sensor.

The final hardware kit is as shown in Fig 4.

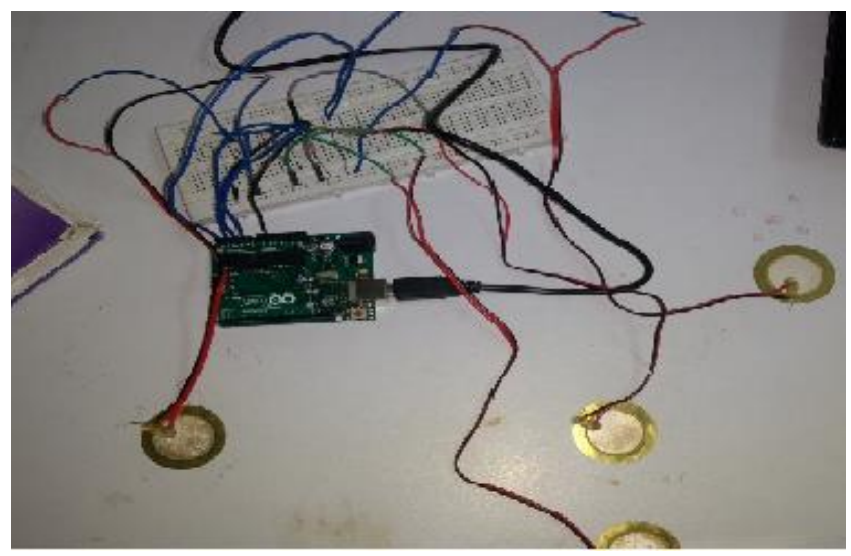

Fig 4: Hardware kit

The medical data from the subjects were taken by grouping the subjects from age of $30-70$ years. The data was collected from 20 healthy subjects and 20 unhealthy subjects who were characterized by traumatic rupture and bone degradation. The data collected from the subjects were entered in the Excel sheet and an average of each piezosensor was calculated. The mean of the healthy subject was calculated and a threshold value was fixed for each sensor. A range was set between each sensor's threshold and based on the threshold they are characterised into Traumatic Rupture or Bone Degradation.

\subsection{Algorithm}

Step 1: The data like Float, Int, String are initiated.

Step 2: The Data entry page is generated to fill in the information like Name, Age, Height, Weight, and Arm Status of the Subjects.

Step 3: The values produced by the vibration of elbow is recorded for each sensor in the form of milli Volts $(\mathrm{mV})$ for every $10 \mathrm{sec}-$ onds in the interval of 120 seconds each.

Step 4: The average of each sensor for the 12 values is calculated.

Step 5: A range is set for each sensor for three conditions i.e., healthy, trauma, degradation using if.... else statement. This displays the result of the subject.

\section{Results and Discussion}

Classifying bone as healthy and unhealthy bone and also the cause of unhealthiness as traumatic rupture and bone degradation has been found from the study based on threshold value. The arduino and the processing software provided with algorithm which extracts data from healthy and unhealthy bone to characterize the cartilage degradation in them. Thus data logging is done through processing, to obtain the required output in excel sheet. The threshold value is fixed by taking the average of 20 healthy and unhealthy subjects separately using piezo sensor .(Table 1)

Table 1: Threshold Values

\begin{tabular}{|c|c|c|c|}
\hline Piezosensor & $\begin{array}{c}\text { Healthy } \\
\text { Threshold }\end{array}$ & $\begin{array}{c}\text { Unhealthy(Trauma) } \\
\text { Threshold }\end{array}$ & $\begin{array}{c}\text { Unhealthy } \\
\text { (Bone Degra- } \\
\text { dation) } \\
\text { Threshold }\end{array}$ \\
\hline 1 & 0.0078 & 0.1394 & 0.2109 \\
\hline 2 & 0.1625 & 0.4853 & 1.0832 \\
\hline 3 & 0.0254 & 0.2183 & 0.5164 \\
\hline 4 & 0.0228 & 0.1624 & 0.1068 \\
\hline
\end{tabular}

Thus a comparison of threshold value with the practical data from the subjects gives a real time output. 


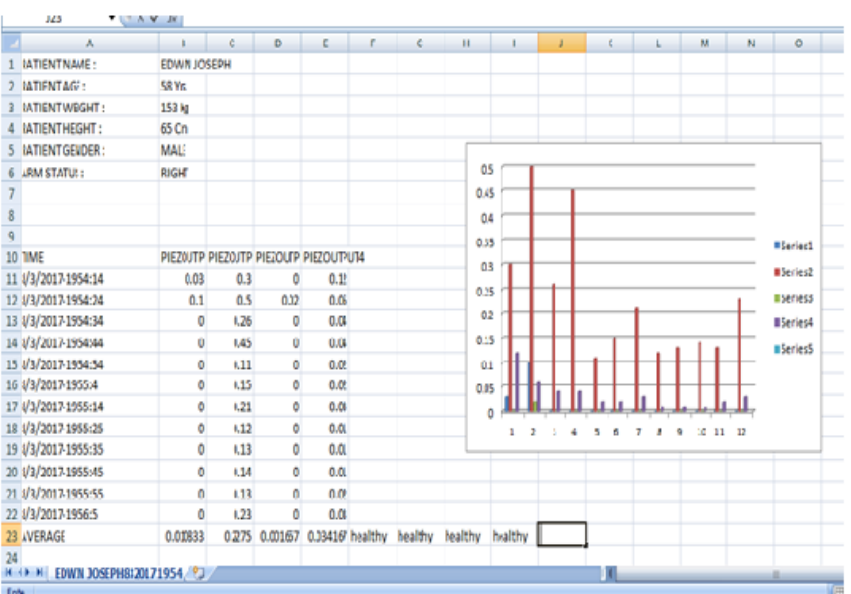

Fig 5: Result of Healthy Subject

The average values obtained from each sensor lies between the threshold values calculated from healthy subject and hence gives result as 'healthy'.(Fig 5) The graphical output represents vibration in each sensor.

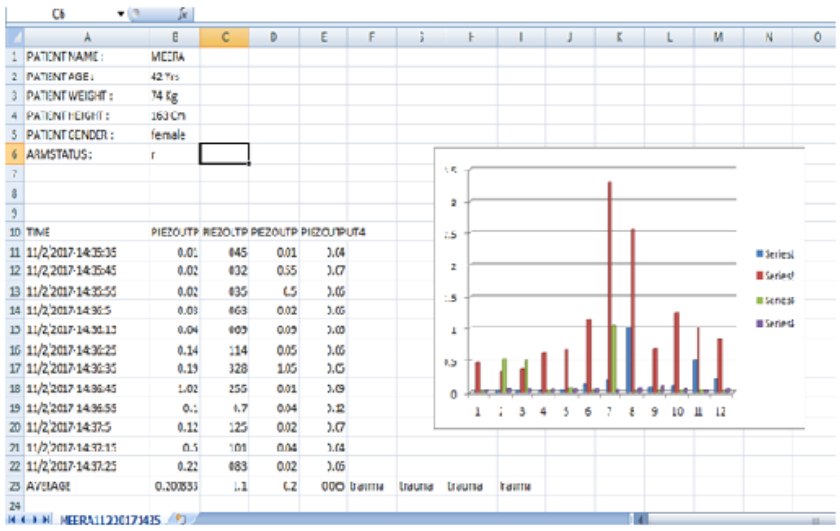

Fig 6: Result of Trauma subject

Thus the average values obtained from each sensor lies between the threshold values calculated from unhealthy subject and hence the result is characterised as 'trauma'.(Fig 6) The graphical output represents vibration in each sensor.

The patient's bone vibration is calculated and results say whether the patient is healthy or unhealthy. Thus depending upon values of bone vibration the status of cartilage degradation can be identified.

\section{Summary}

Using Electroarthrography, the electrical potential at the elbow joint was recorded when a slight load was induced. The threshold for healthy and unhealthy bone was fixed and the unhealthy bone is further being characterized into Trauma or degradation. The vibration between the joint occurs only when there is a gap between them. The gap between them can be created for number of reasons which include bone degradation, cartilage degradation or trauma which leads to misplacement of bones, arthritis, etc. For a healthy person, the vibration between the joints will be very minimal or zero. For a unhealthy person, because of degradation in the cartilage, the vibration between the joints will be more. Comparing these results with the healthy subjects, the threshold values are set.

\section{Conclusion}

With this study the electrical potentials at the surface of the elbow are calculated and they can be characterized into healthy or unhealthy. This technique is easy to find out if the person has defective cartilage or not. The medical method includes X-ray, CT et cetera.

\section{References}

[1] Albert C. Chen, Tara T. Nguyen,Robert L. Sah (1997) "Streaming potentials during the confined compression creep test of normal and proteoglycan-depleted cartilage," Ann. Biomed. Eng., vol. 25 , no. 2, pp. 269-277.

[2] Elsaid KA,Jay GD,Warman ML,Rhee DK,Chichester CO (2005) "Association of articular cartilage degradation and loss of boundary-lubricating ability of synovial fluid following injury and inflammatory arthritis," Arthritis Rheumatism, vol. 52, no. 6, pp. 1746-1755.

[3] Grodzinsky A J, H. Lipshitz \& M. J. Glimcher (1998) "Electromechanical properties of articular cartilage during compression and stress relaxation nature, vol 275,no 5619,pp,448-450.

[4]. Kushner D.( 2011)The making of Arduino. spectrum.ieee.org/geek-life/hands-on/the-making-of-arduino.IEEE Spectrum.

[5]. McMahon P. J and Skinner H. B,(2003)“'Sports medicine," Current Diagnosis Treatment Orthopedics, H. B. Skinner, Ed. New York, NY, USA: Lange Medical Books, pp. 155-173.

[6]. Melvin J.W, 1993) "Fracture Mechanics of Bone ,J.Biomech Eng 115(4B),pp.549- 554

[7] Preville A.M et al.,(2013) “ Electroarthrography: A Novel Method to Assess Articular Cartilage and Diagnose Osteoarthritis by Non-invasive Measurement of Load Induced Electric Potentials at the Surface of the Knee", Osteoarthritis and Cartilage, Vol. 21, pp. 1731- 1737.

[8]. Schmidt-Rohlfing.B, U. Schneider, H. Goost, and J. Silny,( 2011) "Mechanically induced electrical potentials of articular cartilage," J. Biomech., vol. 35, no. 4, pp. 475-482 .

[9]. Quingyi Han, Michael D. Buschmann and Pierre Savard,(2014) " The Forward Problem of Electroarthrography: Modelling Load Induced Electric Potential at the Surface of the Knee" IEEE Transactions on Biomedical Engineering, Vol. 61, no. 7. 